\title{
Supraglottic Laryngeal Paraganglioma: A Rare Entity
}

\author{
Shruti Dhingra ${ }^{1}$, Abhinav Agarwal ${ }^{2}$, Ruchika Juneja ${ }^{3}$
}

\begin{abstract}
Aim and objective: To discuss a rare case of supraglottic paraganglioma presenting as stridor and its management.

Background: Paragangliomas are tumors anatomically arising from the dispersed neuroendocrine system (paraganglia), derived from the neural crest of the autonomic ganglia and characterized by similar neurosecretory cells. They are rarely seen in larynx. Most cases have polypoidal presentation in the endolarynx and diagnosis is usually confirmed after histopathology.

Case description: We present a rare case of laryngeal paraganglioma in which the patient presented with stridor and was successfully treated by open laryngofissure approach leading to excision of the tumor with complete preservation of laryngeal function.

Conclusion: Paragangliomas of the larynx are vascular benign tumors and can have varied presentations. Familiarity with these rare masses is essential for the treating otolaryngologist to prevent an inadvertent biopsy. A carefully selected surgical approach helps preserve adequate laryngeal function and decreases the risk of recurrence.
\end{abstract}

Keywords: Laryngeal paraganglioma, Laryngofissure, Neuroendocrine tumor, Zellballen pattern.

International Journal of Phonosurgery \& Laryngology (2021): 10.5005/jp-journals-10023-1192

\section{INTRODUCTION}

Neuroendocrine neoplasms of the larynx arise from the specialized neuroendocrine cells which are divided into two main groups by Ferlito and Rosai: those of epithelial origin (typical carcinoid, atypical carcinoid, and small cell neuroendocrine carcinoma); and those of neural type (paraganglioma). ${ }^{1}$ Paragangliomas arise from the paraganglion cells derived from the neural crest cells. They are uncommon, slow-growing, and mostly benign tumors. The first case report of laryngeal paraganglioma was given by Blanchard and Saunders in $1955 .^{2}$ They are uncommon, slow-growing, and mostly benign tumors. Less than 80 paragangliomas have been reported in world literature. Thirteen percent of the patients have multiple paragangliomas, carotid body tumors being the most commonly associated form of paraganglioma. ${ }^{3} \mathrm{~A}$ history of smoking along with other predisposing factors in an adult patient may confuse with carcinoma and inadvertently lead to a biopsy of this vascular lesion. We present a rare case of laryngeal paraganglioma and discuss the various approaches toward its surgical management.

\section{Case Description}

A 38-year-old female patient presented to an otolaryngology emergency center with chief complaints of difficulty in breathing for the past year which had exacerbated since the last 5 days and was associated with a high-pitched sound during inspiration. She also complained of gradually progressive hoarseness for the last 2 years. She had been a chronic smoker for the last 10 years. There was no complaint of dysphagia, odynophagia, hemoptysis, or neck swelling. The resident on duty had performed an emergency tracheostomy in view of the respiratory distress and stridor and a biopsy of the lesion was taken at the same time. The examination findings were suggestive of a large, reddish, polypoidal mass arising from the left false vocal cord obscuring the view of bilateral true vocal cords and massive bleeding at the time of biopsy.

The patient was referred to us with the probable diagnosis of cavernous hemangioma. When we performed the flexible laryngoscopy, the mass appeared smaller in size due to the
${ }^{1}$ Department of Otolaryngology and Head Neck Surgery, Maulana Azad Medical College and LNJP Hospital, New Delhi, India

${ }^{2,3}$ Department of Otolaryngology, Maulana Azad Medical College, New Delhi, India

Corresponding Author: Shruti Dhingra, Department of Otolaryngology and Head Neck Surgery, Maulana Azad Medical College and LNJP Hospital, New Delhi, India, Phone: +91 9868453871, e-mail: shrudoc@hotmail.com

How to cite this article: Dhingra S, Agarwal A, Juneja R. Supraglottic Laryngeal Paraganglioma: A Rare Entity. Int J Phonosurg Laryngol 2021;11(1):25-28.

Source of support: Nil

Conflict of interest: None

previously performed biopsy (Fig. 1). Bilateral vocal cords were mobile. Gadolinium-enhanced magnetic resonance imaging (MRI) of the neck (post-biopsy) was suggestive of a homogeneously enhancing soft tissue mass of size $9 \times 5 \mathrm{~mm}$ involving the left false vocal cord causing partial attenuation of airway appearing isointense to mildly hyperintense on $\mathrm{T} 1$ and heterogeneously hyperintense on T2/STIR (Fig. 2). Arteriography and embolization of the lesion were not warranted as it was small in size and well localized. A review of the histopathology slides suggested a paraganglioma. Urinary vanillylmandelic acid, catecholamines, and serum catecholamine levels were within normal limits. The patient was offered the option for undergoing laser excision of the mass at another center, as it was not available at our institute, but the patient refused as she could not afford the cost.

Direct laryngoscopy was performed under general anesthesia before surgery. We chose to operate via the laryngofissure approach as the tumor was subcentimetric and located in the mid-portion of the false vocal cord reaching up to the anterior commissure. Since our patient was tracheostomized and a biopsy had been taken previously, the laryngeal mucosa had already been breached. The tumor was very well visualized by this approach (Fig. 3) and the 


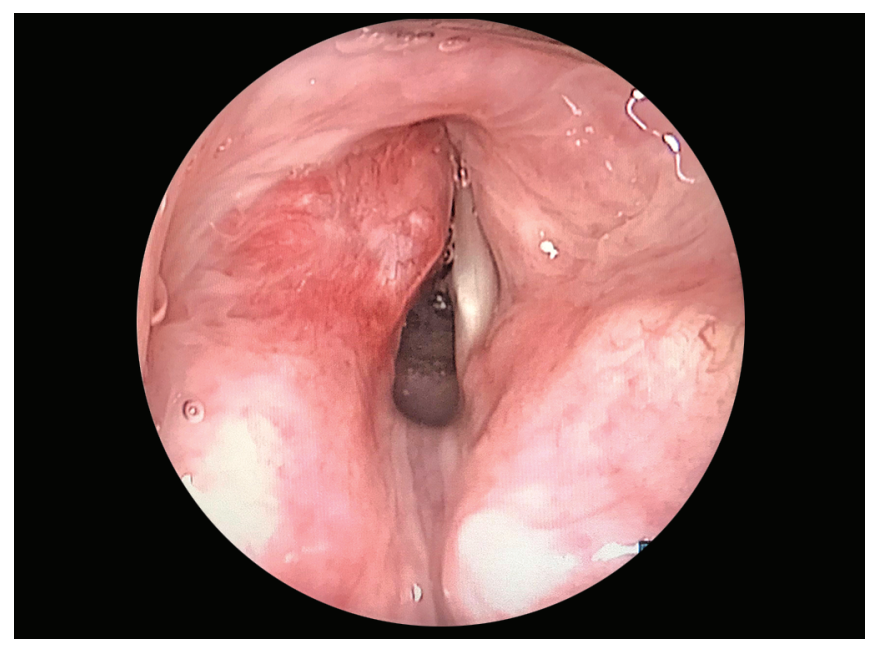

Fig. 1: Reddish, polypoidal mass arising from the left false vocal fold obscuring the view of left true vocal fold and the anterior commissure

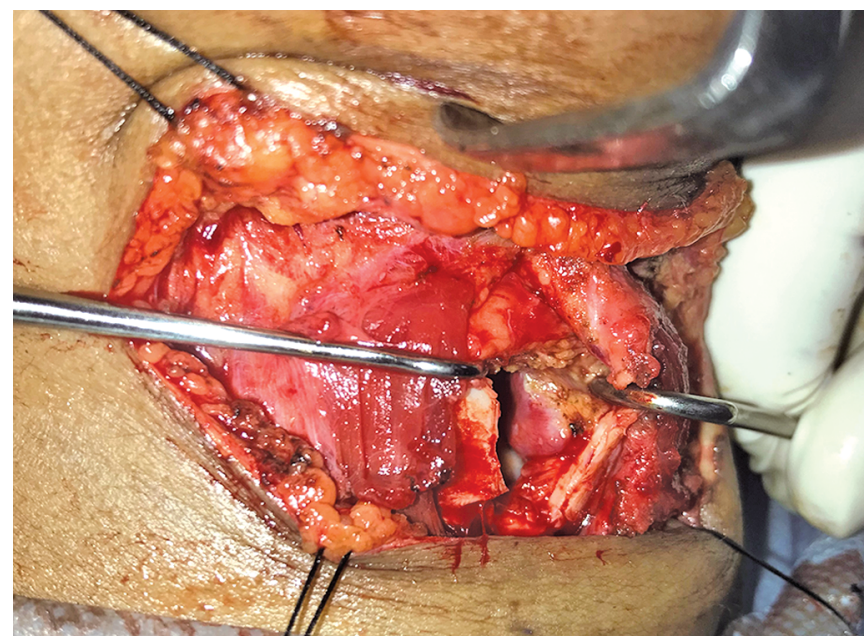

Fig. 3: The mass well visualized in the left false vocal fold, by the laryngofissure approach

mass was completely excised while preserving bilateral true vocal cords (Fig. 4).

Histopathology revealed large polygonal cells with eccentric nuclei and eosinophilic granular cytoplasm. The cells were arranged in nests and a zellballen pattern. On immunohistochemistry, cells showed a strong immunopositivity for synaptophysin, chromogranin, and were negative for cytokeratin. S100 immunostains highlighted the sustentacular cells which confirmed the diagnosis of paraganglioma (Fig. 5).

Postoperatively, the patient recovered well with no complications and a good voice. Bilateral vocal cords were mobile with only minimal slough formation at the false cords at the end of 1 week. She was decannulated 10 days after surgery and discharged. The patient has been on a 1-year follow-up with no recurrence.

\section{Discussion}

Paragangliomas can occur anywhere in the body except the extremities but are most frequently found in the head and neck. The most common sites are the carotid bifurcation and the jugulo-tympanic area. The laryngeal paraganglioma is the only neuroendocrine tumor that is more common in women with a

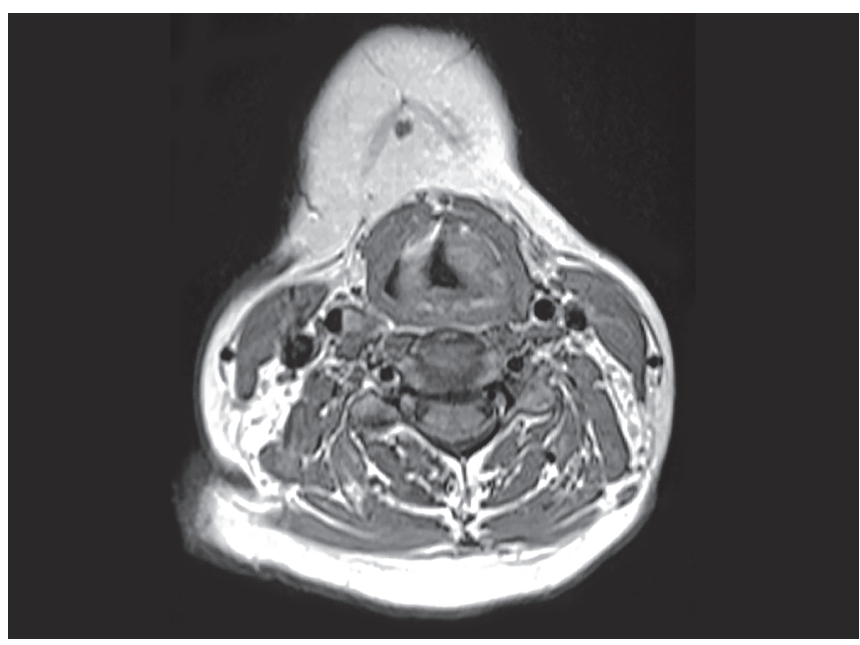

Fig. 2: Contrast-enhanced MRI of the neck showing homogeneously enhancing soft tissue mass of size $9 \times 5 \mathrm{~mm}$ involving the left false vocal cord

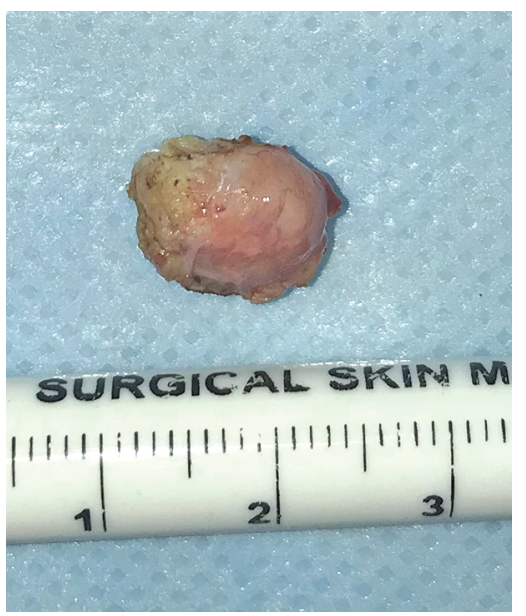

Fig. 4: A well encapsulated $9 \times 5 \mathrm{~mm}$ mass, completely excised

female to male ratio of 3:1 and has a right-sided predilection. ${ }^{3}$ There are two pairs of laryngeal paraganglia. Superior laryngeal paraganglia are located on the edge of the thyroid cartilage. The inferior laryngeal paraganglia lie between the inferior cornua of the thyroid cartilage and the cricoid cartilage. The superior laryngeal paraganglia account for $90 \%$ of laryngeal paragangliomas. Two percent of these tumors have been reported to be malignant but most of these were misdiagnosed as atypical carcinoids. ${ }^{4}$ Hypertension and paraneoplastic syndromes are rarely reported with laryngeal paragangliomas.

Supraglottic paragangliomas can cause hoarseness or chronic cough. Dysphagia, dyspnea, stridor, or a foreign body sensation can be caused by larger size lesions. Subglottic paragangliomas may result in wheezing, stridor, hemoptysis, and vocal fold paralysis. ${ }^{5}$

Because of the highly vascular nature of these neoplasms, a preoperative biopsy often leads to the requirement of a temporary tracheostomy and a possible transfusion. Since these lesions are in the submucosal space, a biopsy breaches the laryngeal mucosa and may lead to incomplete excision. A preoperative presumptive diagnosis of paraganglioma then becomes challenging and contrast-enhanced CT or gadolinium MRI along with four-vessel 


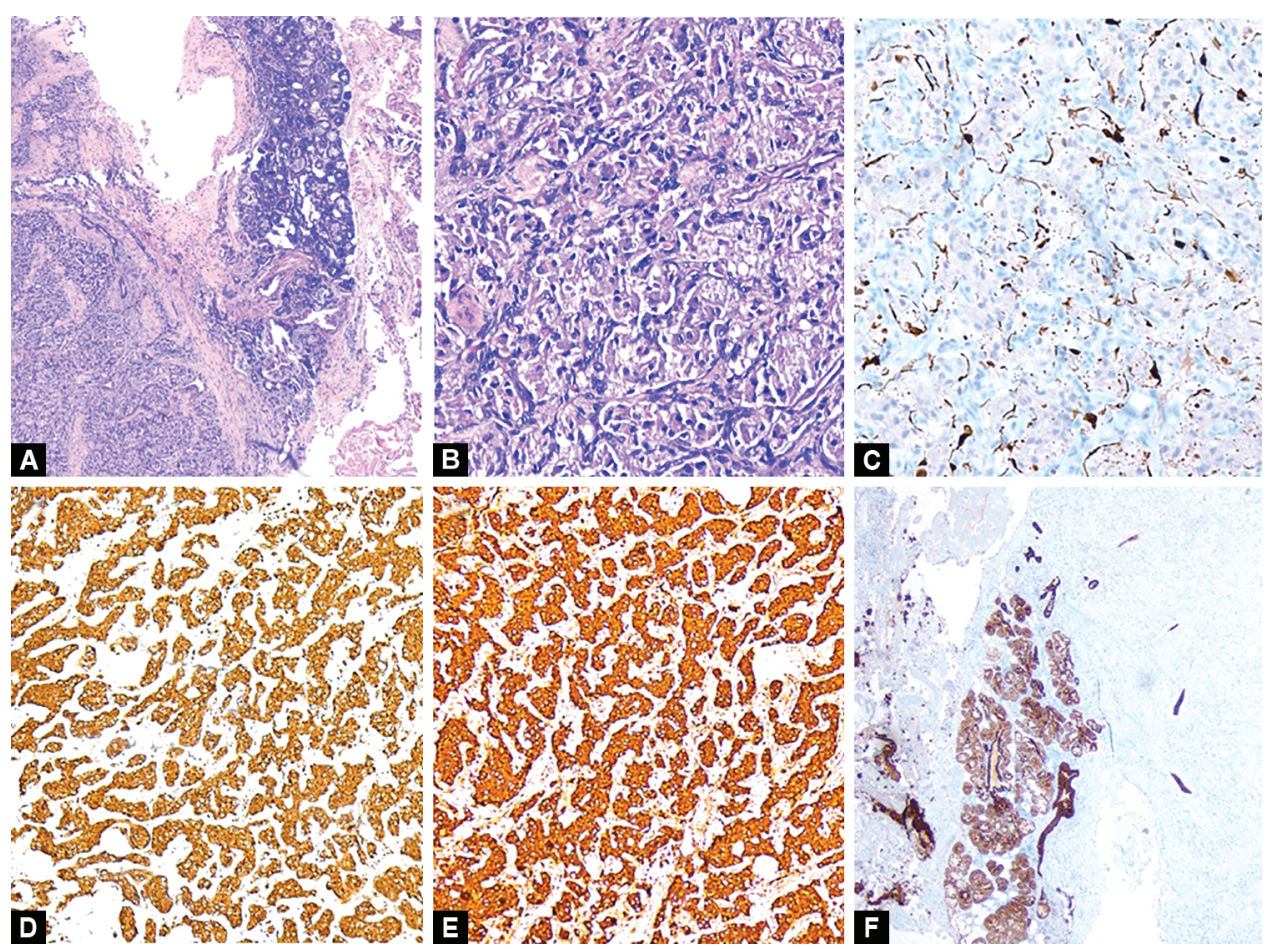

Figs $5 A$ to $F$ : Section showing paraganglioma involving the laryngeal wall $(A, H \& E x 100)$; typical zellballen pattern (B, H\&E x100); $S 100$ immunostain highlighting sustentacular cells (C, DAB x100); Synaptophysin (D) and chromogranin positivity within tumor cells (E) (DAB; x100); pancytokeratin highlighting benign submucosal glands (F, DAB $\times 100)$

arteriography helps in defining the tumor blush and embolizing the feeding vessels besides screening for synchronous lesions. ${ }^{6}$ ${ }^{111}$ Indium pentetreotide octreotide scan has also been suggested by some authors as it increases the sensitivity for detecting small paragangliomas due to the high density of somatostatin type II receptors on the cell surface. ${ }^{7}$ Whereas typical carcinoid, atypical carcinoid, and medullary thyroid carcinoma enhance on octreotide scan, the primary and secondary malignant melanomas and small cell neuroendocrine carcinomas do not enhance.

Histopathologically, it is important to differentiate paragangliomas from other neuroendocrine tumors, especially moderately differentiated neuroendocrine carcinomas, because of the more aggressive nature of the latter and its metastatic potential. The characteristic "Zellballen" arrangement with eosinophilic cytoplasm and large nuclei of tumor cells on histology is generally pathognomonic for paraganglioma as a "Zellballen" pattern has, however, morphologic mimicry can also be seen in occasional carcinoid tumors, melanomas, and medullary carcinomas of the thyroid. Immunohistochemistry can be useful in challenging cases. ${ }^{8}$

The laryngeal paraganglioma is essentially a benign tumor and the treatment remains surgical management with the aim of eradication of tumor with maximal preservation of laryngeal function. We propose that the approach to excision of paragangliomas of the larynx should depend on the size of the tumor and its location.

Endoscopic removal with the cold steel method has been used in small lesions. This approach increases the risk of intraoperative hemorrhage and incomplete excision and most of these patients require revision surgery., ${ }^{9,10}$ Microlaryngeal surgery with carbon dioxide laser would be ideal for small localized lesions with adequate control of bleeding and maximum laryngeal mucosal preservation. However, some authors have reported limited success as cases were eventually converted to open surgery to completely excise the tumor. ${ }^{11,12}$ Large paragangliomas and posteriorly located paragangliomas (involving the aryepiglottic fold and pyriform sinus mucosa) warrant an open surgical approach. A lateral thyrotomy would be ideal for posteriorly located paragangliomas which helps preserve the integrity of the laryngeal mucosa leaving an intact endolarynx, thus avoiding the need for tracheostomy, preventing postoperative dysphagia, and allowing an early discharge. A supraglottic partial laryngectomy would be ideal for large lesions (usually $>3 \mathrm{~cm}$ ) which are difficult to completely expose endoscopically when malignancy is suspected, or synchronous lesions are seen in the neck. This approach helps to remove a margin of normal surrounding tissue, and the feeding vessel usually the superior laryngeal artery or the superior thyroid artery can be ligated in the neck at the time of surgery. Thyrotomy with laryngofissure approach is ideal for more anteriorly placed large supraglottic lesions as it provides an excellent visualization with the larynx being opened up like a book. It achieves a complete removal of the tumor, and voice and swallowing function is preserved. The long-term follow-up with this approach has shown no significant recurrences. ${ }^{13}$

Elective neck dissection is not usually indicated as laryngeal paragangliomas are not known to metastasize to cervical lymph nodes. ${ }^{14}$ Radiotherapy has been considered in a very limited number of cases such as a malignant tumor with intracranial metastases, multisite paraganglioma, and patients unsuitable for surgery. ${ }^{15}$

\section{CONCLUSION}

Paragangliomas of the larynx are benign vascular lesions. Their clinical presentation mimics most of the commonly occurring supraglottic and subglottic lesions. History of smoking and 
presentation with stridor can be confused most often with malignant lesions. Immunohistochemistry remains the diagnostic modality of choice. A correct surgical technique is imperative to preserve laryngeal function.

\section{References}

1. Ferlito A, Rosai J. Terminology and classification of neuroendocrine neoplasms of the larynx. ORL J Otorhinolaryngol Relat Spec 1991;53(4):185/7. DOI: 10.1159/000276217.

2. Blanchard $\mathrm{CL}$, Saunders $\mathrm{WH}$. Chemodectoma of the larynx. Case report. Arch Otolaryngol 1955;61(4):472-474. DOI: 10.1001/ archotol.1955.00720020488016.

3. Ramadan O. Laryngeal paraganglioma: a review article. Otolaryngol Open J 2017;SE(6):S1-S10. DOI: 10.17140/OTLOJ-SE-6-101.

4. Myssiorek D, Rinaldo A, Barnes $L$, et al. Laryngeal paraganglioma: an updated critical review. Acta Otolaryngol 2004;124(9):995-999. DOI: 10.1080/00016480410017576.

5. Hinojar AG, Ramón Prieto J, Muñoz E, et al. Relapsing paraganglioma of the inferior laryngeal paraganglion: case report and review of the literature. Head Neck 2002;24(1):95-102. DOI: 10.1002/hed.1156.

6. Lustrin ES, Palestro C, Vaheesan K. Radiographic evaluation and assessment of paragangliomas. Otolaryngol Clin North Am 2001;34(5):881-906. DOI: 10.1016/S0030-6665(05)70353-4.

7. Smolarz JR, Hanna EY, Williams MD, et al. Paraganglioma of the endolarynx: a rare tumor in an uncommon location. Head Neck Oncol 2010;2(1):2. DOI: 10.1186/1758-32842-2.
8. Milroy CM, Rode J, Moss E. Laryngeal paragangliomas and neuroendocrine carcinomas. Histopathology 1991;18(3):201-209. DOI: 10.1111/j.1365-2559.1991.tb00827.x.

9. Konowitz PM, Lawson W, Som PM, et al. Laryngeal paraganglioma: update on diagnosis and treatment. Laryngoscope 1988;98(1):40-49. DOI: 10.1288/00005537-198801000-00010.

10. Zikk D, Samuel Y, Jossipohov J, et al. Paraganglioma of the supraglottic larynx. ORL J Otorhinolaryngol Relat Spec 1987;49(5):270-275. DOI: 10.1159/000275949.

11. Thirlwall $A S$, Bailey $C M$, Ramsay $A D$, et al. Laryngeal paraganglioma in five-year-old child--the youngest case ever recorded. J Laryngol Otol 1999;113(1):62-64. DOI: 10.1017/S0022215100 143166.

12. Sesterhenn AM, Folz BJ, Lippert BM, et al. Laser surgical treatment of laryngeal paraganglioma. J Laryngol Otol 2003;117(8):641-646. DOI: 10.1258/002221503768200002.

13. Ferlito A, Barnes L, Wenig BM. Identification, classification, treatment, and prognosis of laryngeal paraganglioma. Review of the literature and eight new cases. Ann Otol Rhinol Laryngol 1994;103(7):525-536. DOI: $10.1177 / 000348949410300705$.

14. Ferlito $A$, Barnes $L$, Rinaldo $A$, et al. A review of neuroendocrine neoplasms of the larynx: update on diagnosis and treatment J Laryngol Otol 1998;112(9):827-834. DOI: 10.1017/S00222151 00141830.

15. Pellitteri PK, Rinaldo A, Myssiorek D, et al. Paragangliomas of the head and neck. Oral Oncol 2004;40(6):563-575. DOI: 10.1016/j oraloncology.2003.09.004. 